\title{
A Parabolic Flow of Almost Balanced Metrics
}

\author{
Masaya Kawamura
}

Abstract. We define a parabolic flow of almost balanced metrics. We show that the flow has a unique solution on compact almost Hermitian manifolds.

\section{Introduction}

In [1], Bedulli and Vezzoni introduced a parabolic flow of balanced metrics and they showed that the flow has a unique short-time solution. In recent years some results concerning geometric flows on complex manifolds have been extended to the almost complex setting such as in 4, 6, 9]. In this paper, we will extend their results in the Hermitian geometry to the almost Hermitian geometry.

Let $M^{n}$ be a complex manifold and let $\omega$ be a fundamental $(1,1)$-form associated to a Hermitian metric $g$ on $M$. In the Kähler geometry, the Calabi flow is a well-known gradient flow of the Calabi functional

$$
\omega \mapsto \int_{M}\left(s_{\omega}\right)^{2} \frac{\omega^{n}}{n !}
$$

as it is restricted to the cohomology class of an initial Kähler metric $\omega_{0}$

$$
C_{\omega_{0}}=\left\{\omega_{0}+\sqrt{-1} \partial \bar{\partial} \phi>0 \mid \phi \in C^{\infty}(M, \mathbb{R})\right\}
$$

where $s_{\omega}$ is the scalar curvature of the metric $\omega$. The Calabi flow is as follows:

$$
\frac{\partial}{\partial t} \omega(t)=\sqrt{-1} \partial \bar{\partial} s_{\omega(t)}, \quad \omega(0)=\omega_{0}
$$

The flow above can be alternatively expressed in terms of positive $(n-1, n-1)$-forms as

$$
\frac{\partial}{\partial t} \varphi(t)=\sqrt{-1} \partial \bar{\partial} *_{t}\left(P_{t} \wedge *_{t} \varphi(t)\right), \quad \varphi(0)=\varphi_{0}
$$

where $P_{t}$ is the Ricci form of $\varphi(t)$ and $\varphi_{0}=* \omega_{0} \omega_{0}$.

Let $\left(M^{2 n}, J\right)$ be a real $2 n$-dimensional compact almost complex manifold and let $g$ be an almost Hermitian metric on $M$. Let $\left\{Z_{r}\right\}$ be an arbitrary local $(1,0)$-frame around Received January 29, 2019; Accepted December 8, 2019.

Communicated by Mu-Tao Wang.

2010 Mathematics Subject Classification. Primary: 53C44; Secondary: 53C55, 53C15.

Key words and phrases. almost Hermitian metric, parabolic evolution equation, Chern connection. 
a fixed point $p \in M$ and let $\left\{\zeta^{r}\right\}$ be the associated coframe. Then the associated real $(1,1)$-form $\omega$ with respect to $g$ takes the local expression $\omega=\sqrt{-1} g_{r \bar{k}} \zeta^{r} \wedge \zeta^{\bar{k}}$. We will sometimes also refer to $\omega$ as to an almost Hermitian metric.

Let $\varphi_{0}$ be a positive closed real $(n-1, n-1)$-form on $M$. We investigate the following parabolic flow of almost balanced structures $\varphi$ on $M$ :

$$
\frac{\partial}{\partial t} \varphi(t)=\sqrt{-1} \partial \bar{\partial} *_{t}\left(P_{t} \wedge *_{t} \varphi(t)\right)+(n-1) \Delta_{B C} \varphi(t), \quad d \varphi(t)=0, \quad \varphi(0)=\varphi_{0},
$$

where

$$
\Delta_{B C}:=\partial \overline{\partial \partial}^{*} \partial^{*}+\bar{\partial}^{*} \partial^{*} \partial \bar{\partial}+\bar{\partial}^{*} \partial \partial^{*} \bar{\partial}+\partial^{*} \overline{\partial \partial}^{*} \partial+\bar{\partial}^{*} \bar{\partial}+\partial^{*} \partial
$$

is the modified Bott-Chern Laplacian (cf. [7]) and $*_{t}$ and $P_{t}$ are the Hodge star operator and the Chern-Ricci form locally given by $P_{i \bar{j}}=g^{k \bar{l}} \Omega_{i \bar{j} k \bar{l}}, \Omega$ is the curvature form of the Chern connection with respect to $g$.

We see that the modified Bott-Chern Laplacian is elliptic. For $x \in M, \xi \in T_{x}^{*} M$, we consider the symbol of the operator in the base $\left(\zeta_{I} \wedge \overline{\zeta_{J}}\right)_{|I|=p,|J|=q}$, where $\left\{\zeta_{I}\right\}_{I}$ is the associated coframe with respect to the local $(1,0)$-frame $\left\{Z_{I}\right\}_{I}$ around $x$. Note that all notations below are same as in [7]. Then we have

$$
\begin{gathered}
\left(\sigma_{\partial \overline{\partial \partial}^{*} \partial^{*}}(x, \xi)\right)_{I, J ; I, J}=\left(\sum_{i \in I}\left|\xi_{i}\right|^{2}\right)\left(\sum_{j \in J}\left|\xi_{j}\right|^{2}\right), \\
\left(\sigma_{\partial \overline{\partial \partial}^{*} \partial^{*}}(x, \xi)\right)_{I, J ; I k \backslash i, J}=(-1)^{\alpha_{i}+\gamma_{k}-1} \varepsilon_{i k} \overline{\xi_{i}} \xi_{k}\left(\sum_{j \in J}\left|\xi_{j}\right|^{2}\right), \\
\left(\sigma_{\partial \overline{\partial \partial}^{*} \partial^{*}}(x, \xi)\right)_{I, J ; I, J \backslash j}=(-1)^{\beta_{j}+\delta_{l}-1} \varepsilon_{j l} \xi_{j} \overline{\xi_{l}}\left(\sum_{i \in I}\left|\xi_{i}\right|^{2}\right), \\
\left(\sigma_{\partial \overline{\partial \partial}^{*} \partial^{*}}(x, \xi)\right)_{I, J ; I k \backslash i, J \backslash j}=(-1)^{\alpha_{i}+\beta_{j}+\gamma_{k}+\delta_{l}} \varepsilon_{i k} \varepsilon_{j l} \overline{\xi_{i}} \xi_{k} \xi_{j} \overline{\xi_{l}} .
\end{gathered}
$$

Notice that we have for $k \notin I$, then $\sigma_{\partial \overline{\partial \partial}^{*} \partial^{*}}\left(x, \zeta_{k}\right)=0$, which tells us that the operator $(\partial \bar{\partial})(\partial \bar{\partial})^{*}+\bar{\partial}^{*} \bar{\partial}+\partial^{*} \partial$ cannot be elliptic. We calculate other terms which contribute the symbol:

$$
\begin{aligned}
\left(\sigma_{\bar{\partial}^{*} \partial^{*} \partial \bar{\partial}}(x, \xi)\right)_{I, J ; I, J} & =\left(\sum_{k \notin I}\left|\xi_{k}\right|^{2}\right)\left(\sum_{l \notin J}\left|\xi_{l}\right|^{2}\right), \\
\left(\sigma_{\bar{\partial}^{*} \partial \partial^{*} \bar{\partial}}(x, \xi)\right)_{I, J ; I, J} & =\left(\sum_{i \in I}\left|\xi_{i}\right|^{2}\right)\left(\sum_{l \notin J}\left|\xi_{l}\right|^{2}\right), \\
\left(\sigma_{\partial^{*} \overline{\partial \partial}^{*} \partial}(x, \xi)\right)_{I, J ; I, J} & =\left(\sum_{k \notin I}\left|\xi_{k}\right|^{2}\right)\left(\sum_{j \in J}\left|\xi_{j}\right|^{2}\right) .
\end{aligned}
$$


In this way, we obtain

$$
\sigma_{\Delta_{B C}}(x, \xi)=|\xi|_{\omega}^{4} \operatorname{Id}_{\Lambda^{p, q} T_{x}^{*} M}
$$

Therefore the operator $\Delta_{B C}$ is a fourth order elliptic operator on $M$ in the almost complex setting.

We introduce the definition of an almost balanced metric.

Definition 1.1. Let $\left(M^{2 n}, J\right)$ be an almost complex manifold. A metric $g$ is called an almost balanced metric on $M$ if $g$ is an almost Hermitian metric whose associated real $(1,1)$-form $\omega=\sqrt{-1} g_{i \bar{j}} \zeta^{i} \wedge \zeta^{\bar{j}}$ satisfies $d\left(\omega^{n-1}\right)=0$. And when an almost Hermitian metric $g$ is almost balanced, the triple $\left(M^{2 n}, J, g\right)$ will be called an almost balanced manifold.

An almost balanced structure can be alternatively regarded as a closed positive real $(n-1, n-1)$-form $\varphi$. We define the Bott-Chern cohomology in almost complex geometry as follows:

$$
H_{B C}(M)=\frac{\operatorname{Ker} d}{\operatorname{Im}(\partial \bar{\partial})} .
$$

Our main result is as follows.

Theorem 1.2. Let $(M, J, g)$ be a compact almost Hermitian manifold and let $\varphi_{0}$ be a closed positive real $(n-1, n-1)$-form on $M$. The flow (1.1) admits a unique solution in the Bott-Chern class of $\left[\varphi_{0}\right]$ defined in a maximal interval $[0, \varepsilon)$ on $M$. Moreover, if the initial structure is Kähler, then (1.1) reduces to the Calabi flow.

This paper is organized as follows: in Section 2, we recall some basic definitions and computations in the almost Hermitian geometry. In the last section, we will prove the main theorem by applying Theorem 2.9. Notice that we assume the Einstein convention omitting the symbol of sum over repeated indexes in whole this paper.

\section{Preliminaries}

2.1. The Nijenhuis tensor of the almost complex structure

Let $M$ be a $2 n$-dimensional smooth differentiable manifold. An almost complex structure on $M$ is an endomorphism $J$ of $T M, J \in \Gamma(\operatorname{End}(T M))$, satisfying $J^{2}=-\operatorname{Id}_{T M}$. The pair $(M, J)$ is called an almost complex manifold. Let $(M, J)$ be an almost complex manifold. We define a bilinear map on $C^{\infty}(M)$ for $X, Y \in \Gamma(T M)$ by

$$
4 N(X, Y):=[J X, J Y]-J[J X, Y]-J[X, J Y]-[X, Y]
$$

which is the Nijenhuis tensor of $J$. The Nijenhuis tensor $N$ satisfies $N(X, Y)=-N(Y, X)$, $N(J X, Y)=-J N(X, Y), N(X, J Y)=-J N(X, Y), N(J X, J Y)=-N(X, Y)$. For 
any $(1,0)$-vector fields $V$ and $W, N(V, W)=-[V, W]^{(0,1)}, N(V, \bar{W})=N(\bar{V}, W)=0$ and $N(\bar{V}, \bar{W})=-[\bar{V}, \bar{W}]^{(1,0)}$ since we have $4 N(V, W)=-2([V, W]+\sqrt{-1} J[V, W])$, $4 N(\bar{V}, \bar{W})=-2([\bar{V}, \bar{W}]-\sqrt{-1} J[\bar{V}, \bar{W}])$. An almost complex structure $J$ is called integrable if $N=0$ on $M$. Giving a complex structure to a differentiable manifold $M$ is equivalent to giving an integrable almost complex structure to $M$. Let $(M, J)$ be an almost complex manifold. A Riemannian metric $g$ on $M$ is called $J$-invariant if $J$ is compatible with $g$, i.e., for any $X, Y \in \Gamma(T M), g(X, Y)=g(J X, J Y)$. In this case, the pair $(J, g)$ is called an almost Hermitian structure. The fundamental 2-form $\omega$ associated to a $J$-invariant Riemannian metric $g$, i.e., an almost Hermitian metric, is determined by, for $X, Y \in \Gamma(T M), \omega(X, Y)=g(J X, Y)$. Indeed we have, for any $X, Y \in \Gamma(T M)$,

$$
\omega(Y, X)=g(J Y, X)=g\left(J^{2} Y, J X\right)=-g(J X, Y)=-\omega(X, Y)
$$

and $\omega \in \Gamma\left(\Lambda^{2} T^{*} M\right)$. We will also refer to the associated real fundamental $(1,1)$-form $\omega$ as an almost Hermitian metric. The form $\omega$ is related to the volume form $d V_{g}$ by $n ! d V_{g}=\omega^{n}$. Let a local $(1,0)$-frame $\left\{Z_{r}\right\}$ on $(M, J)$ with an almost Hermitian metric $g$ and let $\left\{\zeta^{r}\right\}$ be a local associated coframe with respect to $\left\{Z_{r}\right\}$, i.e.,

$$
\zeta^{i}\left(Z_{j}\right)=\delta_{j}^{i}, \quad i, j=1, \ldots, n .
$$

Since $g$ is almost Hermitian, its components satisfy $g_{i j}=g_{\overline{i j}}=0$ and $g_{i \bar{j}}=g_{\bar{j} i}=\bar{g}_{\bar{i} j}$.

With using these local frame $\left\{Z_{r}\right\}$ and coframe $\left\{\zeta^{r}\right\}$, we have

$$
N\left(Z_{\bar{i}}, Z_{\bar{j}}\right)=-\left[Z_{\bar{i}}, Z_{\bar{j}}\right]^{(1,0)}=: N_{\overline{i j}}^{k} Z_{k}, \quad N\left(Z_{i}, Z_{j}\right)=-\left[Z_{i}, Z_{j}\right]^{(0,1)}=\overline{N \frac{k}{i j}} Z_{\bar{k}},
$$

and

$$
N=\frac{1}{2} \overline{N \frac{k}{i j}} Z_{\bar{k}} \otimes\left(\zeta^{i} \wedge \zeta^{j}\right)+\frac{1}{2} N \frac{k}{i j} Z_{k} \otimes\left(\zeta^{\bar{i}} \wedge \zeta^{\bar{j}}\right) .
$$

Let $(M, J, g)$ be an almost Hermitian manifold with $\operatorname{dim}_{\mathbb{R}} M=2 n$. An affine connection $D$ on $T M$ is called almost Hermitian connection if $D g=D J=0$. For the almost Hermitian connection, we have the following lemma (cf. [2,3,8,9]).

Lemma 2.1. Let $(M, J, g)$ be an almost Hermitian manifold with $\operatorname{dim}_{\mathbb{R}} M=2 n$. Then for any given $T^{\mathbb{C}} M$-valued $(1,1)$-form $\Theta=\left(\Theta^{i}\right)_{1 \leq i \leq n}$, there exists a unique almost Hermitian connection $D$ on $(M, J, g)$ such that the $(1,1)$-part of the torsion is equal to the given $\Theta$.

If the (1,1)-part of the torsion of an almost Hermitian connection vanishes everywhere, then the connection is called the second canonical connection or the Chern connection. We will refer the connection as the Chern connection and denote it by $\nabla$.

Now let $\nabla$ be the Chern connection on $M$. We denote the structure coefficients of Lie bracket by

$$
\left[Z_{i}, Z_{j}\right]=: B_{i j}^{r} Z_{r}+B_{i j}^{\bar{r}} Z_{\bar{r}}, \quad\left[Z_{i}, Z_{\bar{j}}\right]=: B_{i \bar{j}}^{r} Z_{r}+B_{i \bar{j}}^{\bar{r}} Z_{\bar{r}}, \quad\left[Z_{\bar{i}}, Z_{\bar{j}}\right]=: B_{\overline{i j}}^{r} Z_{r}+B_{\overline{i j}}^{\bar{r}} Z_{\bar{r}}
$$


Note that $\left[Z_{i}, Z_{j}\right]^{(0,1)}=-\overline{N_{\overline{i j}}^{r}} Z_{\bar{r}},\left[Z_{\bar{i}}, Z_{\bar{j}}\right]^{(1,0)}=-N_{\overline{i j}}^{r} Z_{r}$ and then $B_{i j}^{\bar{r}}=-\overline{N_{\overline{i j}}^{r}}, B_{\overline{i j}}^{r}=-N_{\overline{i j}}^{r}$. Notice that $J$ is integrable if and only if the $B_{i j}^{\bar{r}}$ 's vanish. For instance,

$$
\left[Z_{i}, Z_{\bar{j}}\right]=\left[Z_{i}, Z_{\bar{j}}\right]^{(1,0)}+\left[Z_{i}, Z_{\bar{j}}\right]^{(0,1)},
$$

where

$$
\left[Z_{i}, Z_{\bar{j}}\right]^{(1,0)}=\frac{1}{2}\left(\left[Z_{i}, Z_{\bar{j}}\right]-\sqrt{-1} J\left[Z_{i}, Z_{\bar{j}}\right]\right), \quad\left[Z_{i}, Z_{\bar{j}}\right]^{(0,1)}=\frac{1}{2}\left(\left[Z_{i}, Z_{\bar{j}}\right]+\sqrt{-1} J\left[Z_{i}, Z_{\bar{j}}\right]\right) .
$$

Note that for any $p$-form $\psi$, there holds that (cf. [9])

$$
\begin{aligned}
d \psi\left(X_{1}, \ldots, X_{p+1}\right)= & \sum_{i=1}^{p+1}(-1)^{i+1} X_{i}\left(\psi\left(X_{1}, \ldots, \widehat{X_{i}}, \ldots, X_{p+1}\right)\right) \\
& +\sum_{i<j}(-1)^{i+j} \psi\left(\left[X_{i}, X_{j}\right], X_{1}, \ldots, \widehat{X_{i}}, \ldots, \widehat{X_{j}}, \ldots, X_{p+1}\right) .
\end{aligned}
$$

We directly compute that

$$
d \zeta^{s}=-\frac{1}{2} B_{k l}^{s} \zeta^{k} \wedge \zeta^{l}-B_{k \bar{l}}^{s} \zeta^{k} \wedge \zeta^{\bar{l}}+\frac{1}{2} N \frac{s}{k l} \zeta^{\bar{k}} \wedge \zeta^{\bar{l}} .
$$

According to the direct computation above, we may split the exterior differential operator $d: \Lambda^{p} M \otimes_{\mathbb{R}} \mathbb{C} \rightarrow \Lambda^{p+1} M \otimes_{\mathbb{R}} \mathbb{C}$, into four components

$$
d=A+\partial+\bar{\partial}+\bar{A}
$$

with

$$
\begin{array}{ll}
\partial: \Lambda^{p, q} M \rightarrow \Lambda^{p+1, q} M, & \bar{\partial}: \Lambda^{p, q} M \rightarrow \Lambda^{p, q+1} M, \\
A: \Lambda^{p, q} M \rightarrow \Lambda^{p+2, q-1} M, & \bar{A}: \Lambda^{p, q} M \rightarrow \Lambda^{p-1, q+2} M .
\end{array}
$$

In terms of these components, the condition $d^{2}=0$ can be written as

$$
\begin{gathered}
A^{2}=0, \quad \partial A+A \partial=0, \quad \overline{\partial A}+\bar{A} \bar{\partial}=0, \quad \bar{A}^{2}=0, \\
A \bar{\partial}+\partial^{2}+\bar{\partial} A=0, \quad A \bar{A}+\partial \bar{\partial}+\bar{\partial} \partial+\bar{A} A=0, \quad \partial \bar{A}+\bar{\partial}^{2}+\bar{A} \partial=0 .
\end{gathered}
$$

For any real $(1,1)$-form $\eta=\sqrt{-1} \eta_{i \bar{j}} \zeta^{i} \wedge \zeta^{\bar{j}}$, we have

$$
\begin{aligned}
& \bar{\partial} \eta=\frac{\sqrt{-1}}{2}\left(Z_{\bar{j}}\left(\eta_{k \bar{i}}\right)-Z_{\bar{i}}\left(\eta_{k \bar{j}}\right)-B_{k \bar{i}}^{s} \eta_{s \bar{j}}+B_{k \bar{j}}^{s} \eta_{s \bar{i}}+B_{\overline{i j}}^{\bar{s}} \eta_{k \bar{s}}\right) \zeta^{k} \wedge \zeta^{\bar{i}} \wedge \zeta^{\bar{j}} \\
& \partial \eta=\frac{\sqrt{-1}}{2}\left(Z_{i}\left(\eta_{j \bar{k}}\right)-Z_{j}\left(\eta_{i \bar{k}}\right)-B_{i j}^{s} \eta_{s \bar{k}}-B_{i \bar{k}}^{\bar{s}} \eta_{j \bar{s}}+B_{j \bar{k}}^{\bar{s}} \eta_{i \bar{s}}\right) \zeta^{i} \wedge \zeta^{j} \wedge \zeta^{\bar{k}}
\end{aligned}
$$

From these computations above, we have

$$
\begin{aligned}
\bar{\partial} \omega & =\frac{\sqrt{-1}}{2}\left(Z_{\bar{j}}\left(g_{k \bar{i}}\right)-Z_{\bar{i}}\left(g_{k \bar{j}}\right)-B_{k \bar{i}}^{s} g_{s \bar{j}}+B_{k \bar{j}}^{s} g_{s \bar{i}}+B \frac{\bar{s}}{i j} g_{k \bar{s}}\right) \zeta^{k} \wedge \zeta^{\bar{i}} \wedge \zeta^{\bar{j}} \\
& =\frac{\sqrt{-1}}{2} T_{\overline{j i} k} \zeta^{k} \wedge \zeta^{\bar{i}} \wedge \zeta^{\bar{j}}
\end{aligned}
$$


and

$$
\begin{aligned}
\partial \omega & =\frac{\sqrt{-1}}{2}\left(Z_{i}\left(g_{j \bar{k}}\right)-Z_{j}\left(g_{i \bar{k}}\right)-B_{i j}^{s} g_{s \bar{k}}-B_{i \bar{k}}^{\bar{s}} g_{j \bar{s}}+B_{j \bar{k}}^{\bar{s}} g_{i \bar{s}}\right) \zeta^{i} \wedge \zeta^{j} \wedge \zeta^{\bar{k}} \\
& =\frac{\sqrt{-1}}{2} T_{i j \bar{k}} \zeta^{i} \wedge \zeta^{j} \wedge \zeta^{\bar{k}}
\end{aligned}
$$

where $T_{\overline{j i k}}=\overline{T_{j i \bar{k}}}$.

A direct computation yields for any $\varphi \in C^{\infty}(M, \mathbb{R})$,

$$
\begin{gathered}
(d J d \varphi)\left(Z_{i}, Z_{j}\right)=-2 \sqrt{-1}\left[Z_{i}, Z_{j}\right]^{(0,1)} \varphi, \quad(d J d \varphi)\left(Z_{\bar{i}}, Z_{\bar{j}}\right)=2 \sqrt{-1}\left[Z_{\bar{i}}, Z_{\bar{j}}\right]^{(1,0)} \varphi, \\
(d J d \varphi)\left(Z_{i}, Z_{\bar{j}}\right)=2 \sqrt{-1}\left(Z_{i} Z_{\bar{j}}-\left[Z_{i}, Z_{\bar{j}}\right]^{(0,1)}\right) \varphi .
\end{gathered}
$$

Hence, especially we have

$$
\sqrt{-1} \partial \bar{\partial} \varphi=\frac{1}{2}(d J d \varphi)^{(1,1)}=\sqrt{-1}\left(Z_{i} Z_{\bar{j}}-\left[Z_{i}, Z_{\bar{j}}\right]^{(0,1)}\right) \varphi \zeta^{i} \wedge \zeta^{\bar{j}} .
$$

2.2. The torsion and the curvature on almost complex manifolds

Since the Chern connection $\nabla$ preserves $J$, we have

$$
\nabla_{i} Z_{j}=\Gamma_{i j}^{r} Z_{r}, \quad \nabla_{i} Z_{\bar{j}}=\Gamma_{i \bar{j}}^{\bar{r}} Z_{\bar{r}}
$$

where

$$
\Gamma_{i j}^{r}=g^{r \bar{s}} Z_{i}\left(g_{j \bar{s}}\right)-g^{r \bar{s}} g_{j \bar{l}} B_{i \bar{s}}^{\bar{l}}, \quad \Gamma_{i p}^{p}=Z_{i}(\log \operatorname{det} g)-B_{i \bar{s}}^{\bar{s}} .
$$

We can obtain that $\Gamma_{i \bar{j}}^{\bar{r}}=B_{i \bar{j}}^{\bar{r}}$ since the $(1,1)$-part of the torsion of the Chern connection vanishes everywhere (cf. [5]).

Note that the mixed derivatives $\nabla_{i} Z_{\bar{j}}$ do not depend on $g$ (cf. 8 ). Let $\left\{\gamma_{j}^{i}\right\}$ be the connection form, which is defined by $\gamma_{j}^{i}=\Gamma_{s j}^{i} \zeta^{s}+\Gamma_{\bar{s} j}^{i} \zeta^{\bar{s}}$. The torsion $T$ of the Chern connection $\nabla$ is defined by $T^{i}:=d \zeta^{i}-\zeta^{p} \wedge \gamma_{p}^{i}, T^{\bar{i}}:=d \zeta^{\bar{i}}-\zeta^{\bar{p}} \wedge \gamma_{\bar{p}}^{\bar{i}}$, which has no $(1,1)$-part and the only non-vanishing components are as follows:

$$
T_{i j}^{s}=\Gamma_{i j}^{s}-\Gamma_{j i}^{s}-B_{i j}^{s}, \quad T_{i j}^{\bar{s}}=-B_{i j}^{\bar{s}} .
$$

These equations tell us that $T$ splits into $T=T^{\prime}+T^{\prime \prime}$, where $T^{\prime} \in \Gamma\left(\Lambda^{2,0} M \otimes T^{1,0} M\right)$ and $T^{\prime \prime} \in \Gamma\left(\Lambda^{2,0} M \otimes T^{0,1} M\right)$. We also lower the index of torsion and denote it by $T_{i j \bar{k}}=T_{i j}^{s} g_{s \bar{k}}$. Note that $T^{\prime \prime}$ depends only on $J$ and it can be regarded as the Nijenhuis tensor of $J$, that is, $J$ is integrable if and only if $T^{\prime \prime}$ vanishes.

We denote by $\Omega$ the curvature of the Chern connection $\nabla$. We can regard $\Omega$ as a section of $\Lambda^{2} M \otimes T M, \Omega \in \Gamma\left(\Lambda^{2} M \otimes T M\right)$ and $\Omega$ splits in $\Omega=H+R+\bar{H}$, where 
$R \in \Gamma\left(\Lambda^{1,1} M \otimes \Lambda^{1,1} M\right), H \in \Gamma\left(\Lambda^{2,0} M \otimes \Lambda^{1,1} M\right)$. The curvature form can be expressed by $\Omega_{j}^{i}=d \omega_{j}^{i}+\omega_{s}^{i} \wedge \omega_{j}^{s}$. In terms of $Z_{r}$ 's, we have

$$
\begin{aligned}
& R_{i \bar{j} k}^{r}=\Omega_{k}^{r}\left(Z_{i}, Z_{\bar{j}}\right)=Z_{i}\left(\Gamma_{\bar{j} k}^{r}\right)-Z_{\bar{j}}\left(\Gamma_{i k}^{r}\right)+\Gamma_{i s}^{r} \Gamma_{\bar{j} k}^{s}-\Gamma_{\bar{j} s}^{r} \Gamma_{i k}^{s}-B_{i \bar{j}}^{s} \Gamma_{s k}^{r}+B_{\bar{j} i}^{\bar{s}} \Gamma_{\bar{s} k}^{r}, \\
& H_{i j k}^{r}=\Omega_{k}^{r}\left(Z_{i}, Z_{j}\right)=Z_{i}\left(\Gamma_{j k}^{r}\right)-Z_{j}\left(\Gamma_{i k}^{r}\right)+\Gamma_{i s}^{r} \Gamma_{j k}^{s}-\Gamma_{j s}^{r} \Gamma_{i k}^{s}-B_{i j}^{s} \Gamma_{s k}^{r}-B_{i j}^{\bar{s}} \Gamma_{\bar{s} k}^{r}, \\
& H_{\overline{i j k}}^{r}=\Omega_{k}^{r}\left(Z_{\bar{i}}, Z_{\bar{j}}\right)=Z_{\bar{i}}\left(\Gamma_{\bar{j} k}^{r}\right)-Z_{\bar{j}}\left(\Gamma_{\bar{i} k}^{r}\right)+\Gamma_{\bar{i} s}^{r} \Gamma_{\bar{j} k}^{s}-\Gamma_{\bar{j} s}^{r} \Gamma_{\bar{i} k}^{s}-B_{\overline{i j}}^{s} \Gamma_{s k}^{r}-B_{\overline{i j}}^{\bar{s}} \Gamma_{\bar{s} k}^{r},
\end{aligned}
$$

and we deduce that by using $\Gamma_{k p}^{p}=Z_{k}(\log \operatorname{det} g)-B_{k \bar{p}}^{\bar{p}}$,

$$
\begin{gathered}
P_{i \bar{j}}=-\partial_{i} \partial_{\bar{j}} \log \operatorname{det} g+Z_{\bar{j}}\left(B_{i \bar{r}}^{\bar{r}}\right)+Z_{i}\left(B_{\bar{j} r}^{r}\right)+B_{i \bar{j}}^{s} B_{s \bar{r}}^{\bar{r}}-B_{i \bar{j}}^{\bar{s}} B_{\bar{s} r}^{r} \\
R_{i j}=\left[Z_{i}, Z_{j}\right]^{(0,1)}(\log \operatorname{det} g)-Z_{i}\left(B_{j \bar{r}}^{\bar{r}}\right)+Z_{j}\left(B_{i \bar{r}}^{\bar{r}}\right)+B_{i j}^{s} B_{s \bar{r}}^{\bar{r}}-B_{i j}^{\bar{s}} B_{\bar{s} r}^{r}
\end{gathered}
$$

and

$$
R_{\overline{i j}}=-\left[Z_{\bar{i}}, Z_{\bar{j}}\right]^{(1,0)}(\log \operatorname{det} g)+Z_{\bar{i}}\left(B_{\bar{j} r}^{r}\right)-Z_{\bar{j}}\left(B_{\bar{i} r}^{r}\right)+B_{\overline{i j}}^{s} B_{s \bar{r}}^{\bar{r}}-B_{\overline{i j}}^{\bar{s}} B_{\bar{s} r}^{r} .
$$

The Chern-Ricci form $\operatorname{Ric}(\omega)$ is defined by

$$
\operatorname{Ric}(\omega)=\frac{\sqrt{-1}}{2} R_{k l} \zeta^{k} \wedge \zeta^{l}+\sqrt{-1} P_{k \bar{l}} \zeta^{k} \wedge \zeta^{\bar{l}}+\frac{\sqrt{-1}}{2} R_{\overline{k l}} \zeta^{\bar{k}} \wedge \zeta^{\bar{l}} .
$$

It is a closed real 2 -form. If $J$ is integrable, it is a closed real $(1,1)$-form. If furthermore, $J$ is integrable and $d \omega=0$, then the Chern-Ricci form coincides with the Ricci form defined by the Levi-Civita connection of $\omega$.

\subsection{Hodge star operator and Hodge system}

A Hodge star operator $*: \Lambda^{p, q} M \rightarrow \Lambda^{n-q, n-p} M$ defined by

$$
\alpha \wedge * \bar{\beta}=g(\alpha, \bar{\beta}) \frac{\omega^{n}}{n !} .
$$

Note that $* \omega=\frac{1}{(n-1) !} \omega^{n-1}$. Let $\mathcal{Q}: \Lambda_{+}^{1,1} M \rightarrow \Lambda^{n-1, n-1} M$ be the smooth map $\omega \mapsto *_{\omega} \omega$, where let $\Lambda_{+}^{n-1, n-1} M=\mathcal{Q}\left(\Lambda_{+}^{1,1} M\right)$ be the set of positive $(n-1, n-1)$-forms. Let us fix $\omega \in \Lambda_{+}^{1,1} M$. Then $\Lambda^{1,1} M$ splits as

$$
\Lambda^{1,1} M=\mathbb{C} \omega \oplus \Lambda_{0}^{1,1} M
$$

where $\Lambda_{0}^{1,1} M=\left\{\sigma \in \Lambda^{1,1} M \mid \sigma \wedge \omega^{n-1}=0\right\}$ is the set of primitive (1,1)-forms. Similarly, $\Lambda^{n-1, n-1} M$ splits as

$$
\Lambda^{n-1, n-1} M=\mathbb{C} \varphi \oplus \Lambda_{0}^{n-1, n-1} M,
$$

where $\varphi=* \omega$ and $\Lambda_{0}^{n-1, n-1} M=\left\{\gamma \in \Lambda^{n-1, n-1} M \mid \gamma \wedge \omega=0\right\}$.

We introduce the following algebraic lemma. 
Lemma 2.2. 1, Lemma 2.1] Let $\sigma$ be in $\Lambda_{0}^{1,1} M$, then for $0 \leq k \leq n-2$,

$$
*\left(\sigma \wedge \omega^{k}\right)=-\frac{1}{(n-2-k) !} \sigma \wedge \omega^{n-2-k} .
$$

In particular, if $h=h_{1} \omega+h_{0} \in \Lambda^{1,1} M$, then

$$
* h=\frac{1}{(n-1) !} h_{1} \omega^{n-1}-\frac{1}{(n-2) !} h_{0} \wedge \omega^{n-2} .
$$

Let $M^{2 n}$ be an almost complex manifold. We denote $\Lambda^{p, q} M$ the bundle of complex forms of type $(p, q)$ on $M$ and $C^{\infty}\left(M, \Lambda^{p, q} M\right)$ the vector space of its smooth sections. The following fiber bundles $\Lambda_{+}^{1,1} M, \Lambda_{+}^{n-1, n-1} M$ are naturally defined. An almost balanced structure on $M$ is a global coclosed section $\omega$ of $\Lambda_{+}^{1,1} M$, or equivalently, is a closed section $\varphi$ of $\Lambda_{+}^{n-1, n-1} M$.

By using Lemma 2.1, we can obtain the following result.

Lemma 2.3. 1, Lemma 2.5] Assume

$$
\frac{d}{d t} \varphi=h_{1} \varphi+*_{\varphi} h_{0}
$$

with $h_{1} \in C^{\infty}(M, \mathbb{R})$ and $h_{0} \in C^{\infty}\left(M, \Lambda_{0}^{1,1} M\right)$. Then

$$
\frac{d}{d t}\left(*_{\varphi} \varphi\right)=\frac{h_{1}}{n-1} *_{\varphi} \varphi-h_{0}
$$

Let $\Delta_{A}$ denote the modified Aeppli Laplacian (cf. [7]), which is defined by

$$
\Delta_{A}:=\bar{\partial}^{*} \partial^{*} \partial \bar{\partial}+\partial \overline{\partial \partial}^{*} \partial^{*}+\partial \bar{\partial}^{*} \bar{\partial} \partial^{*}+\bar{\partial} \partial^{*} \partial \bar{\partial}^{*}+\partial \partial^{*}+\overline{\partial \partial}^{*}
$$

Although we see that the operator $(\partial \bar{\partial})^{*}(\partial \bar{\partial})+\partial \partial^{*}+\overline{\partial \partial}^{*}$ cannot be elliptic, we can show that the modified Aeppli Laplacian $\Delta_{A}$ is a fourth order elliptic operator in the same way for proving that $\Delta_{B C}$ is elliptic in Introduction of this paper. We will need a Hodge-like decomposition induced by $\Delta_{A}$. We can prove the Aeppli decomposition based on the result that $\Delta_{A}$ is elliptic.

Proposition 2.4. (cf. [7]) If $(M, J, g)$ is a compact almost Hermitian manifold, then we have the following orthogonal decomposition for every $(p, q)$,

$$
C^{\infty}\left(M, \Lambda^{p, q}\right)=\mathcal{H}_{\Delta_{A}}^{p, q}(M) \oplus(\operatorname{Im} \partial+\operatorname{Im} \bar{\partial}) \oplus \operatorname{Im}(\partial \bar{\partial})^{*}
$$

where $\mathcal{H}_{\Delta_{A}}^{p, q}(M)=\operatorname{Ker} \Delta_{A}$.

We prepare the following lemma for proving the following proposition. 
Lemma 2.5. 4, Lemma 3.4] One has

$$
\partial \bar{\partial} \partial=0, \quad \partial \bar{\partial}^{2}=0 .
$$

The following property is a crucial step in the proof of Theorem 1.2 . The proof is similar to the one in [1, Proposition 2.4]. The difference appears only in the part of using Lemma 2.5 and the following lemma. By applying some results in the proof of [4. Lemma 3.4], we further obtain the following equalities.

Lemma 2.6. One has

$$
\partial^{2} \bar{\partial}=0, \quad \bar{\partial} \partial \bar{\partial}=0 .
$$

Proof. By using $A \bar{A}+\partial \bar{\partial}+\bar{\partial} \partial+\bar{A} A=0, \partial \bar{A}+\bar{\partial}^{2}+\bar{A} \partial=0, \partial A+A \partial=0$ and the results in the proof of $\left[4\right.$, Lemma 3.4]; $\partial \bar{A} A=0, A \bar{\partial}^{2}=-A \bar{A} \partial$ and $\partial \bar{\partial} \partial=0$, we obtain

$$
\begin{aligned}
\partial^{2} \bar{\partial} & =\partial \partial \bar{\partial}=-\partial(A \bar{A}+\bar{\partial} \partial+\bar{A} A)=-\partial A \bar{A}-\partial \bar{\partial} \partial-\partial \bar{A} A \\
& =A \partial \bar{A}=-A \bar{\partial}^{2}-A \bar{A} \partial=0 .
\end{aligned}
$$

Proposition 2.7. (cf. [1, Proposition 2.4]) Let $G_{A}$ be the Green operator associated to the modified Aeppli Laplacian $\Delta_{A}$. Then for every $\psi \in \partial \bar{\partial} C^{\infty}\left(M, \Lambda^{p, q}\right)$, we have

$$
\psi=\partial \bar{\partial} G_{A}(\partial \bar{\partial})^{*}(\psi) .
$$

Proof. Choose arbitrary $\psi \in \partial \bar{\partial} \Lambda^{p, q}$. By applying the Aeppli decomposition and $u \in$ $\mathcal{H}_{\Delta_{A}}^{p, q}(M) \Leftrightarrow \bar{\partial}^{*} u=\partial^{*} u=\partial \bar{\partial} u=0$ (cf. 7]), and $\partial \bar{\partial} \partial=0, \partial \bar{\partial}^{2}=0$ in Lemma 2.5, we have $\psi=\partial \bar{\partial} \beta$ with $\beta \in \operatorname{Im}(\partial \bar{\partial})^{*}$. Especially, since we have $\partial^{*} \bar{\partial}^{*} \partial^{*}=0, \bar{\partial}^{*} \bar{\partial}^{*} \partial^{*}=0$ from Lemmas 2.5 and 2.6 , we have $\Delta_{A} \beta=(\partial \bar{\partial})^{*} \partial \bar{\partial} \beta$, which tells us that

$$
\beta=G_{A}\left((\partial \bar{\partial})^{*} \partial \bar{\partial} \beta\right)=G_{A}\left((\partial \bar{\partial})^{*} \psi\right),
$$

and hence we obtain

$$
\psi=\partial \bar{\partial} G_{A}(\partial \bar{\partial})^{*}(\psi)
$$

Next we introduce a Hodge system.

Definition 2.8. A Hodge system on a manifold $M$ consists of the following sequence

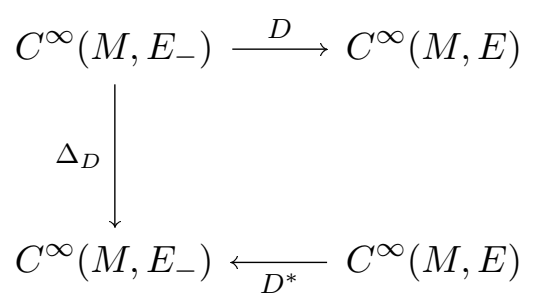

where $E_{-}$and $E$ are fiber bundles over $M$ with an assigned metric along their fibers, $D$ is a differential operator, $D^{*}$ is the formal adjoint of $D$ and $\Delta_{D}$ is an elliptic operator such that $\psi=D G D^{*} \psi$ for every $\psi \in \operatorname{Im} D$, where $G$ is the Green operator of $\Delta_{D}$. 
Consider a Hodge system on a compact manifold $M$ as in Definition 2.8, Let $O$ be an open subset of $E$ such that $\pi(O)=M$, where $\pi: E \rightarrow M$ is the projection. Consider a non-linear partial differential operator of order $2 m$

$$
L: C^{\infty}(M, O) \rightarrow C^{\infty}(M, E)
$$

and a fixed initial datum $\varphi_{0} \in C^{\infty}(M, O)$ such that

$$
L\left(\varphi_{0}+D \gamma\right) \in \operatorname{Im} D
$$

for every $\gamma \in C^{\infty}\left(M, E_{-}\right)$. We consider the following evolution problem

$$
\frac{\partial}{\partial t} \varphi(t)=L(\varphi(t)), \quad \varphi(0)=\varphi_{0}
$$

where $\varphi(t)$ is in the following space

$$
U=\left\{\varphi_{0}+D \gamma \mid \gamma \in C^{\infty}\left(M, E_{-}\right)\right\} \cap C^{\infty}(M, O)
$$

and $\varphi(t)$ is required to depend smoothly on time.

Let $\mathcal{D}^{2 m}(E, E)$ denote the space of partial differential operators on $E$ of order $\leq 2 m$, which can be seen as the space of smooth sections of a vector bundle. A linear partial operator $Q$ of order $2 m$ is said to be strongly elliptic if its principal symbol $\sigma_{Q}(x, \xi)$ satisfies the following inequality:

$$
-\left\langle\sigma_{Q}(x, \xi) v, v\right\rangle_{E} \geq \lambda|\xi|^{2 m}|v|^{2 m}
$$

for some positive constant $\lambda$ and for all $(x, \xi) \in T M, \xi \neq 0$ and $v \in E_{x}$, whose definition dose not depend on the metric $\langle\cdot, \cdot\rangle_{E}$ along the fibers on $E$. The principal symbol of $Q$ is defined by

$$
\sigma_{Q}(x, \xi) v=\frac{(\sqrt{-1})^{2 m}}{(2 m) !} Q\left(f^{2 m} u\right)(x)
$$

for $f \in C^{\infty}(M)$ with $f(x)=0, d_{x} f=\xi$ and $u \in C^{\infty}(E)$ with $u(x)=v$. We denote by $\left.L_{*}\right|_{\varphi}$ the derivative of the operator $L$ at $\varphi$.

Theorem 2.9. [1, Theorem 3.2] Let $\left(E_{-}, E, D, \Delta_{D}\right)$ be a Hodge system on a compact Riemannian manifold $M$. Let $L, \varphi_{0}$ and $U$ be as above. Assume that there exists a nonlinear partial differential operator

$$
\widetilde{L}: C^{\infty}(M, O) \rightarrow \mathcal{D}^{2 m}(E, E), \quad \varphi \mapsto \widetilde{L}_{\varphi}
$$

such that

(1) $\widetilde{L}_{\varphi}$ is strong elliptic for every $\varphi \in U$; 
(2) $\left.L_{*}\right|_{\varphi}(\psi)=\widetilde{L}_{\varphi}(\psi)$ for every $\varphi \in U$ and $\psi \in D C^{\infty}\left(M, E_{-}\right)$.

Assume further that

$$
\left.L_{*}\right|_{\varphi}(D \theta)=D l_{\varphi}(\theta)
$$

for every $\theta \in C^{\infty}\left(M, E_{-}\right)$, where $l_{\varphi}$ is a strongly elliptic linear differential operator on $E_{-}$. Then there exists $\varepsilon>0$ such that the system (2.1) has a unique solution $\varphi \in C^{\infty}([0, \varepsilon), U)$.

\section{Proof of Theorem 1.2}

We consider

$$
E_{-}=\Lambda_{\mathbb{R}}^{n-2, n-2} M \stackrel{D=\sqrt{-1} \partial \bar{\partial}}{\longrightarrow} E=\Lambda_{\mathbb{R}}^{n-1, n-1} M,
$$

where $\Lambda_{\mathbb{R}}^{p, p} M$ is the bundle of real $(p, p)$-forms; the subset $U$ is the set of smooth sections of $\Lambda_{+}^{n-1, n-1} M$ lying in the same cohomology class as $\varphi_{0}$. Let

$$
L: C^{\infty}\left(M, \Lambda_{+}^{n-1, n-1} M\right) \rightarrow C^{\infty}\left(M, \Lambda_{\mathbb{R}}^{n-1, n-1} M\right)
$$

be the operator $L(\varphi)=\sqrt{-1} \partial \bar{\partial} *(P \wedge * \varphi)+(n-1) \Delta_{B C} \varphi$.

The Chern Ricci-type curvature $P_{i \bar{j}}$ is locally given as follows:

$$
P_{i \bar{j}}=-\partial_{i} \partial_{\bar{j}} \log G+Z_{\bar{j}}\left(B_{i \bar{r}}^{\bar{r}}\right)+Z_{i}\left(B_{\bar{j} r}^{r}\right)+B_{i \bar{j}}^{s} B_{s \bar{r}}^{\bar{r}}-B_{i \bar{j}}^{\bar{s}} B_{\bar{s} r}^{r},
$$

where $G$ is the determinant of the matrix $g=\left(g_{i \bar{j}}\right)$. In the following, $\dot{\alpha}$ will denote the derivative with respect to time of the tensor $\alpha$.

Lemma 3.1. The derivative of $P$ is

$$
\dot{P}=-\sqrt{-1} \partial \bar{\partial}(\omega, \dot{\omega})
$$

where $(\cdot, \cdot)$ denotes the pointwise scalar product of $(1,1)$-forms.

Proof. We derive in $t=t_{0}$ and we fix a unitary $(1,0)$-frame with respect to $g\left(t_{0}\right)$. Then we have

$$
\begin{aligned}
\dot{P}_{i \bar{j}} & =-\partial_{i} \partial_{\bar{j}} \frac{\partial}{\partial t} \log G+Z_{\bar{j}}\left(\frac{\partial}{\partial t} B_{i \bar{r}}^{\bar{r}}\right)+Z_{i}\left(\frac{\partial}{\partial t} B_{\bar{j} r}^{r}\right)+\frac{\partial}{\partial t}\left(B_{i \bar{j}}^{s} B_{s \bar{r}}^{\bar{r}}-B_{i \bar{j}}^{\bar{s}} B_{\bar{s} r}^{r}\right) \\
& =-\partial_{i} \partial_{\bar{j}} \frac{\partial}{\partial t} \log G,
\end{aligned}
$$

where we used that $B_{i \bar{j}}^{s}=-B_{\bar{j} i}^{s}$, $B_{i \bar{j}}^{\bar{s}}$ 's do not depend on $t$, which depend only on $J$ (cf. 8, Lemma 5.2]) because that the mixed derivatives $\nabla_{i} Z_{\bar{j}}$ do not depend on $g$. Since we have $\frac{\partial}{\partial t} \log G=\operatorname{tr}\left(g^{-1} \dot{g}\right)$ and $\operatorname{tr}\left(g^{-1} \dot{g}\right)=(\omega, \dot{\omega})$, we obtain the desired equation. 
Now, we shall prove that for every closed $\varphi \in C^{\infty}\left(M, \Lambda_{+}^{n-1, n-1} M\right)$ and every closed $\psi \in C^{\infty}\left(M, \Lambda_{\mathbb{R}}^{n-1, n-1} M\right)$, we have

$$
\left.L_{*}\right|_{\varphi}(\psi)=(1-n) \Delta_{B C} \psi+\sqrt{-1} \partial \bar{\partial} \Psi_{\varphi}(\psi)
$$

where $\Psi_{\varphi}$ is a linear algebraic operator on $\psi$ with coefficients depending on the torsion of $\varphi$ in a universal way. We denote the operator $L$ by $L=S+T$, where

$$
S(\varphi)=\sqrt{-1} \partial \bar{\partial} *(P \wedge * \varphi), \quad T(\varphi)=(n-1) \Delta_{B C} \varphi
$$

Let $\psi=\frac{\partial}{\partial t} \varphi$ be a tangent vector to $U$ at $\varphi$. Then we may write $\psi=h_{1} \varphi+* h_{0}$, where $h_{1}$ is a smooth function on $M$ and $h_{0}$ is a section of $\Lambda_{0}^{1,1} M$. Note that $h_{1}=\frac{1}{n}(\omega, \dot{\omega})$. The derivative of $S$ is obtained by using Lemma 3.1 as follows:

$$
\left.S_{*}\right|_{\varphi}(\psi)=\sqrt{-1} \partial \bar{\partial} *(\dot{P} \wedge * \varphi)+\sqrt{-1} \partial \bar{\partial} \Phi_{1}(\psi)=n \partial \bar{\partial} *\left(\partial \bar{\partial} h_{1} \wedge * \varphi\right)+\sqrt{-1} \partial \bar{\partial} \Phi_{1}(\psi),
$$

where $\Phi_{1}$ is an algebraic operator depending on $\varphi$ in a universal way. The derivative of $T$ is, from Lemma 2.3 ,

$$
\begin{aligned}
\left.T_{*}\right|_{\varphi}(\psi) & =\left.T_{*}\right|_{\varphi}\left(h_{1} \varphi+* h_{0}\right) \\
& =-\partial \bar{\partial} *\left(\partial \bar{\partial} h_{1} \wedge * \varphi+(1-n)(\partial \bar{\partial}+A \bar{A}+\bar{A} A) h_{0}\right)+\sqrt{-1} \partial \bar{\partial} \Phi_{2}(\psi)
\end{aligned}
$$

for a suitable linear zeroth order operator $\Phi_{2}$, where we used that $\bar{\partial} \partial=-\partial \bar{\partial}-A \bar{A}-\bar{A} A$ and $A h_{1}=\bar{A} h_{1}=0$. By combining these, we have

$$
\left.L_{*}\right|_{\varphi}(\psi)=(n-1) \partial \bar{\partial} *\left(\partial \bar{\partial} h_{1} \wedge * \varphi+(\partial \bar{\partial}+A \bar{A}+\bar{A} A) h_{0}\right)+\sqrt{-1} \partial \bar{\partial} \Phi_{1}(\psi)+\sqrt{-1} \partial \bar{\partial} \Phi_{2}(\psi) .
$$

Since $\psi$ is a $d$-closed $(n-1, n-1)$-form, we have $0=d \psi=(A+\partial+\bar{\partial}+\bar{A}) \psi=(\partial+\bar{\partial}) \psi$, which tells us that we obtain $\partial \psi=\bar{\partial} \psi=0$, and hence we get

$$
\begin{aligned}
\left(\Delta_{B C}\right)_{\varphi} \psi & =\partial \overline{\partial \partial}^{*} \partial^{*} \psi=\partial \overline{\partial \partial}^{*} \partial^{*}\left(h_{1} \varphi+* h_{0}\right) \\
& =-\partial \bar{\partial} *\left(\partial \bar{\partial} h_{1} \wedge * \varphi+(\partial \bar{\partial}+A \bar{A}+\bar{A} A) h_{0}\right)+\sqrt{-1} \partial \bar{\partial} \Phi_{3}(\psi) .
\end{aligned}
$$

Therefore, we have

$$
\left.L_{*}\right|_{\varphi}(\psi)=-(n-1)\left(\Delta_{B C}\right)_{\varphi} \psi+\sqrt{-1} \partial \bar{\partial} \Phi_{\varphi}(\psi),
$$

where $\Phi_{\varphi}=\Phi_{1}+\Phi_{2}-(n-1) \Phi_{3}$. Thus we obtained the equation (3.1). Recall that it can be shown that $-\Delta_{B C}$ is strongly elliptic as we see in Section 1 . We finally consider

$$
l_{\varphi}=-(n-1)\left(\Delta_{A}\right)_{\varphi}+\sqrt{-1} \Phi_{\varphi} \circ \partial \bar{\partial} .
$$

As we have confirmed that $-\Delta_{A}$ is strongly elliptic. In addition, we have the following 
Proposition 3.2. One has

$$
\Delta_{B C} \partial \bar{\partial}=\partial \bar{\partial} \Delta_{A}
$$

Proof. By using Lemma 2.6, we obtain

$$
\begin{aligned}
\Delta_{B C} \partial \bar{\partial} & =\left(\partial \overline{\partial \partial}^{*} \partial^{*}+\bar{\partial}^{*} \partial^{*} \partial \bar{\partial}+\bar{\partial}^{*} \partial \partial^{*} \bar{\partial}+\partial^{*} \overline{\partial \partial}^{*} \partial+\bar{\partial}^{*} \bar{\partial}+\partial^{*} \partial\right) \partial \bar{\partial} \\
& =\partial \overline{\partial \partial}^{*} \partial^{*} \partial \bar{\partial}+\bar{\partial}^{*} \partial^{*} \partial \bar{\partial} \partial \bar{\partial}+\bar{\partial}^{*} \partial \partial^{*} \bar{\partial} \partial \bar{\partial}+\partial^{*} \overline{\partial \partial}^{*} \partial^{2} \bar{\partial}+\bar{\partial}^{*} \bar{\partial} \partial \bar{\partial}+\partial^{*} \partial^{2} \bar{\partial} \\
& =\partial \overline{\partial \partial}^{*} \partial^{*} \partial \bar{\partial} .
\end{aligned}
$$

On the other hand, using Lemma 2.5 we have

$$
\begin{aligned}
\partial \bar{\partial} \Delta_{A} & =\partial \bar{\partial}\left(\bar{\partial}^{*} \partial^{*} \partial \bar{\partial}+\partial \overline{\partial \partial}^{*} \partial^{*}+\partial \bar{\partial}^{*} \bar{\partial} \partial^{*}+\bar{\partial} \partial^{*} \partial \bar{\partial}^{*}+\partial \partial^{*}+\overline{\partial \partial}^{*}\right) \\
& =\partial \bar{\partial}^{*} \partial^{*} \partial \bar{\partial}+\partial \bar{\partial} \partial \bar{\partial}^{*} \partial^{*}+\partial \bar{\partial} \partial \bar{\partial}^{*} \bar{\partial} \partial^{*}+\partial \bar{\partial}^{2} \partial^{*} \partial \bar{\partial}^{*}+\partial \bar{\partial} \partial \partial^{*}+\partial \bar{\partial}^{2} \bar{\partial}^{*} \\
& =\partial \bar{\partial}^{*} \partial^{*} \partial \bar{\partial}
\end{aligned}
$$

Hence we get $\Delta_{B C} \partial \bar{\partial}=\partial \bar{\partial} \Delta_{A}$.

Then we have that $\left.L_{*}\right|_{\varphi}(D \psi)=D l_{\varphi}(\psi)$ for every closed $\psi \in C^{\infty}\left(M, \Lambda_{\mathbb{R}}^{n-2, n-2} M\right)$. Now we may apply Theorem 2.9 and therefore we conclude that the flow (1.1) admits a unique solution defined a maximal interval $[0, \varepsilon)$ for some $\varepsilon>0$. If $\varphi_{0}$ is the $(n-1, n-1)$ positive form of a Kähler structure, then the solution $\varphi_{0}+\beta(t)$ to 1.1 corresponds to a family of Kähler forms $\omega(t)$ solving the Calabi flow (cf. [1]). This completes the proof of Theorem 1.2 .

\section{References}

[1] L. Bedulli and L. Vezzoni, A parabolic flow of balanced metrics, J. Reine Angew. Math. 723 (2017), 79-99.

[2] S.-s. Chern, Characteristic classes of Hermitian manifolds, Ann. of Math. (2) 47 (1946), 85-121.

[3] P. Gauduchon, Hermitian connections and Dirac operators, Boll. Un. Mat. Ital. B (7) 11 (1997), no. 2, suppl., 257-288.

[4] M. Kawamura, An almost pluriclosed flow, Geome. Flows 2 (2017), no. 1, 72-85.

[5] _ Parabolic flows on almost Hermitian manifolds, Kyushu J. Math. 73 (2019), no. $1,69-87$.

[6] _ A scalar Calabi-type flow in the almost Hermitian geometry, Tsukuba J. Math. 43 (2019), no. 1, 37-54. 
[7] M. Schweitzer, Autour de la cohomologie de Bott-Chern, arXiv:0709.3528v1.

[8] L. Vezzoni, On Hermitian curvature flow on almost complex manifolds, Differential Geom. Appl. 29 (2011), no. 5, 709-722.

[9] T. Zheng, An almost complex Chern-Ricci flow, J. Geom. Anal. 28 (2018), no. 3, 2129-2165.

Masaya Kawamura

Department of Social Design Engineering, National Institute of Technology, Kochi College, 200-1, Otsu, Monobe, Nankoku, Kochi, 783-8508 Japan

E-mail address: kawamura@kochi-ct.ac.jp 